\title{
Notch Signaling and Ghost Cell Fate in the Calcifying Cystic Odontogenic Tumor
}

\author{
C. H. Siar ${ }^{1}$, T. Kawakami², R. R. Buery³, K. Nakano' , M. Tomida ${ }^{4}$, H. Tsujigiwa ${ }^{3}$, P. P. Han ${ }^{5}$, \\ H. Nagatsuka ${ }^{3}$ and K. H. $\mathrm{Ng}^{6}$ \\ ${ }^{1}$ Department of Oral Pathology, Oral Medicine \& Periodontology, Faculty of Dentistry, University of Malaya, Kuala Lumpur, Malaysia \\ ${ }^{2}$ Hard Tissue Pathology Unit, Matsumoto Dental University Graduate School of Oral Medicine, Shiojiri, Japan \\ ${ }^{3}$ Department of Oral Pathology and Medicine, Graduate School of Medicine, Dentistry \& Pharmaceutical Science, Okayama University, \\ Okayama, Japan \\ ${ }^{4}$ Department of Oral Physiology, Matsumoto Dental University School of Dentistry, Shiojiri, Japan \\ ${ }^{5}$ School of Dentistry, University of Southern California, Los Angeles, CA, USA \\ ${ }^{6}$ formerly Unit of Stomatology, Cancer Research Centre, Institute for Medical Research, Jalan Pahang, Kuala Lumpur, Malaysia
}

\begin{abstract}
Notch signaling is an evolutionarily conserved mechanism that enables adjacent cells to adopt different fates. Ghost cells (GCs) are anucleate cells with homogeneous pale eosinophilic cytoplasm and very pale to clear central areas (previous nucleus sites). Although GCs are present in a variety of odontogenic lesions notably the calcifying cystic odontogenic tumor (CCOT), their nature and process of formation remains elusive. The aim of this study was to investigate the role of Notch signaling in the cell fate specification of GCs in CCOT. Immunohistochemical staining for four Notch receptors (Notch1, Notch2, Notch3 and Notch4) and three ligands (Jagged1, Jagged2 and Delta1) was performed on archival tissues of five CCOT cases. Level of positivity was quantified as negative $(0)$, mild $(+)$, moderate $(2+)$ and strong $(3+)$. Results revealed that GCs demonstrated overexpression for Notch1 and Jagged1 suggesting that Notch1Jagged1 signaling might serve as the main transduction mechanism in cell fate decision for GCs in CCOT. Protein localizations were largely membranous and/or cytoplasmic. Mineralized GCs also stained positive implicating that the calcification process might be associated with upregulation of these molecules. The other Notch receptors and ligands were weak to absent in GCs and tumoral epithelium. Stromal endothelium and fibroblasts were stained variably positive.
\end{abstract}

Key words: Notch, Jagged, Delta, Calcifying cystic odontogenic tumor (CCOT), Ghost cells

\section{INTRODUCTION}

Notch signaling is an evolutionarily conserved mechanism that enables adjacent cells to adopt different fates [1]. The Drosophila Notch gene encodes a transmembrane receptor with a large extracellular domain carrying multiple epidermal growth factor-like repeats and a cytoplasmic domain required for signal transduction [1]. In vertebrates, there are four Notch recep- tor proteins (Notch1, Notch2, Notch3, and Notch4) and five membrane-bound ligand proteins (Delta1, Delta2, Delta4, Jagged1, and Jagged2) [1]. Signals exchanged between neighboring cells through binding of ligand with its cognate receptor initiates short range events including differentiation, proliferation, and apoptotic events at all stages of development, thus controlling organ formation and morphogenesis [1]. Deregulation of Notch signaling has been implicated in developmental abnormalities and neoplasias [2].

Ghost cells are large pale anucleate cells with homogeneous pale eosinophilic cytoplasm and very pale to clear central areas instead of a basophilic nucleus [3]. They tend to form small clusters or large masses. Although characteristic of calcifying cystic odontogenic tumors (CCOT) [4], ghost cells are also found in other odontogenic lesions namely ameloblastoma [5] odontoma [6] and ameloblastic fibro-odontoma [7], and in nonodontogenic tumors such as pilomatrixoma [8], a tumor with hair matrix cell differentiation, and craniopharyngioma, a tumor of the pituitary gland [9]. Several theories of ghost cell formation have been put forth including that these cells are most likely abnormal keratinized bodies, or they might represent simple cell degeneration or a form of enamel matrix; or might be apoptotic odontogenic cells or represent different stages of normal and abnormal keratin formation resulting from metaplastic transformation of odontogenic tumors [4]. The World Health Organization Classification of Head and Neck Tumors considered ghost cells as transitory squamous cells at various stages of development [10]. However to date the true nature of these ghost cells remains unresolved. The calcifying process of ghost cells also remains ill-understood.

Odontogenic tumors form a special research interest in this region because these neoplasms represent a clinically significant group of jaw tumors that are both challenging to diagnose and treat. Our group has worked on the demographic and immunoprofile of some of these tumors in the hope of gaining a better 
understanding on the development and progression of these neoplasms [11-23]. In recent years, we have focused our attention on the oncogenic role of Notch signaling in the tumorigenesis of ameloblastomas [1920], squamous odontogenic tumor [21], calcifying epithelial odontogenic tumor [22] and CCOT [23]. In the latter study which was based on a single case report, we observed that Notch1 positivity was detected in both the epithelial and ectomensenchymal tumoral components suggesting that this molecule might play some roles in the cytological differentiation and acquisition of tissue-specific characteristics. These preliminary findings prompted us to examine a further five new cases of CCOT for both Notch receptor (Notch1-4) and ligand (Jagged1-2 and Delta1) activity specifically to clarify the role of Notch signaling in the fate of ghost cells.

\section{MATERIALS AND Methods}

\section{Tissue Samples}

The test sample consisted of five CCOT cases that fulfilled the World Health Organization diagnostic criteria [10]. These were obtained from patients (four female and one male) whose ages ranged from 13-55 years (mean age: 23.5 years). All cases were intrabony in location ( 3 mandibular and two maxillary lesions) and presented as cystic CCOT characterized by ameloblastomatous lining epithelium, ghost cells and calcifications [6].
From the archival formalin-fixed, paraffin-embedded tissue blocks of these cases, new five micron thick sections were cut for staining with hematoxylin-eosin and Congo red, and for immunohistochemistry with primary antibodies directed against Notch (1-4), Jagged(1-2) and Delta 1 (Table 1).

\section{IMMUNOHISTOCHEMISTRY}

Immunostaining was performed using the Envision technique as previously described $[19,20]$. Briefly, pretreatment of deparaffinized sections for antigen retrieval was done by microwaving $\left(99^{\circ} \mathrm{C}\right)$ in $10 \mathrm{nM}$ of citrate buffer ( $\mathrm{pH}$ 6, $20 \mathrm{~min}$ ). For blocking endogenous peroxidase, the sections were immersed in $0.3 \%$ methanol containing 3\% hydrogen peroxide for 20 min and rinsing in $0.05 \mathrm{M}$ Tris-buffered saline (TBS) ( 5 min, two times) before immersing in blocking solution (Dako Corporation, Carpintera, CA, USA) for 20 $\mathrm{min}$ at room temperature. Subsequently the sections were incubated with the primary antibody (Table 1) for $1 \mathrm{~h}$ at room temperature. Immunoreactions were performed using the Envision Kit (Dako Corporation, Carpintera, CA, USA). The antigenic sites were visualized using diaminobenzidine (DAB) substrate chromogen (Dako Corporation, Carpintera, CA, USA) and counterstained with Mayer's hematoxylin. Appropriate positive controls were applied. For negative control, sections were treated as above but without the primary antibody. All the control sections were negative.

Table 1. Antibodies, sources and dilutions.

\begin{tabular}{lllll}
\hline Antibody & Clonality & Catalogue ID & Source & Dilution \\
\hline Notch1 & Mouse monoclonal & LS-C16925 & LifeSpan BioSciences Inc., Seattle, WA, USA & $1: 500$ \\
Notch2 & Rabbit polyclonal & LS-B3399 & LifeSpan BioSciences Inc., Seattle, WA, USA & $1: 500$ \\
Notch3 & Rabbit polyclonal & LS-B1621 & LifeSpan BioSciences Inc., Seattle, WA, USA & $1: 500$ \\
Notch4 & Rabbit polyclonal & LS-C40785 & LifeSpan BioSciences Inc., Seattle, WA, USA & $1: 500$ \\
Jagged1 & Rabbit polyclonal & LS-B2442 & LifeSpan BioSciences Inc., Seattle, WA, USA & $1: 500$ \\
Jagged2 & Rabbit polyclonal & Ab60041 & Abcam Inc., Cambridge, MA, USA & $1: 500$ \\
Delta1 & Rabbit polyclonal & LS-B72 & LifeSpan BioSciences Inc., Seattle, WA, USA & $1: 500$ \\
\hline
\end{tabular}

Table 2. Immunoexpression of Notch receptors and ligand proteins in calcifying cystic odontogenic tumors $(\mathrm{n}=5)$.

\begin{tabular}{lccccccc}
\hline CCOT components & Notch1 & Notch2 & Notch3 & Notch4 & Jagged1 & Jagged2 & Delta1 \\
\hline Basal pre-ameloblast-like cells & + & - & - & + & - & - & - \\
Suprabasal stellate reticulum-like cells & + & - & - & + & + & - & - \\
Transitory squamous cells & + & - & - & + & + & - & - \\
Ghost cells & $2+$ & - & - & + & $3+$ & - & - \\
Calcifications & + & - & + & + & + & - & - \\
Connective tissue stroma & - & - & + & + & - & - & - \\
Dentinoid & NA & NA & NA & NA & NA & NA & NA \\
Odontome & NA & NA & NA & NA & NA & NA & NA \\
\hline
\end{tabular}

CCOT, Calcifying cystic odontogenic tumor; -, negative; + , mild $(<25 \%$ positivity); $2+$, moderate $(25-50 \%$ positivity); $3+$, strong $(>50 \%$ positivity); NA, Not available 


\section{IMMUNOHISTOCHEMICAL ANALYSIS}

Notch receptor and ligand protein distribution pattern and levels of staining intensity in all five CCOT cases were evaluated using descriptive and semiquantitative methods $(19,20)$. In the latter, the immunostained sec-
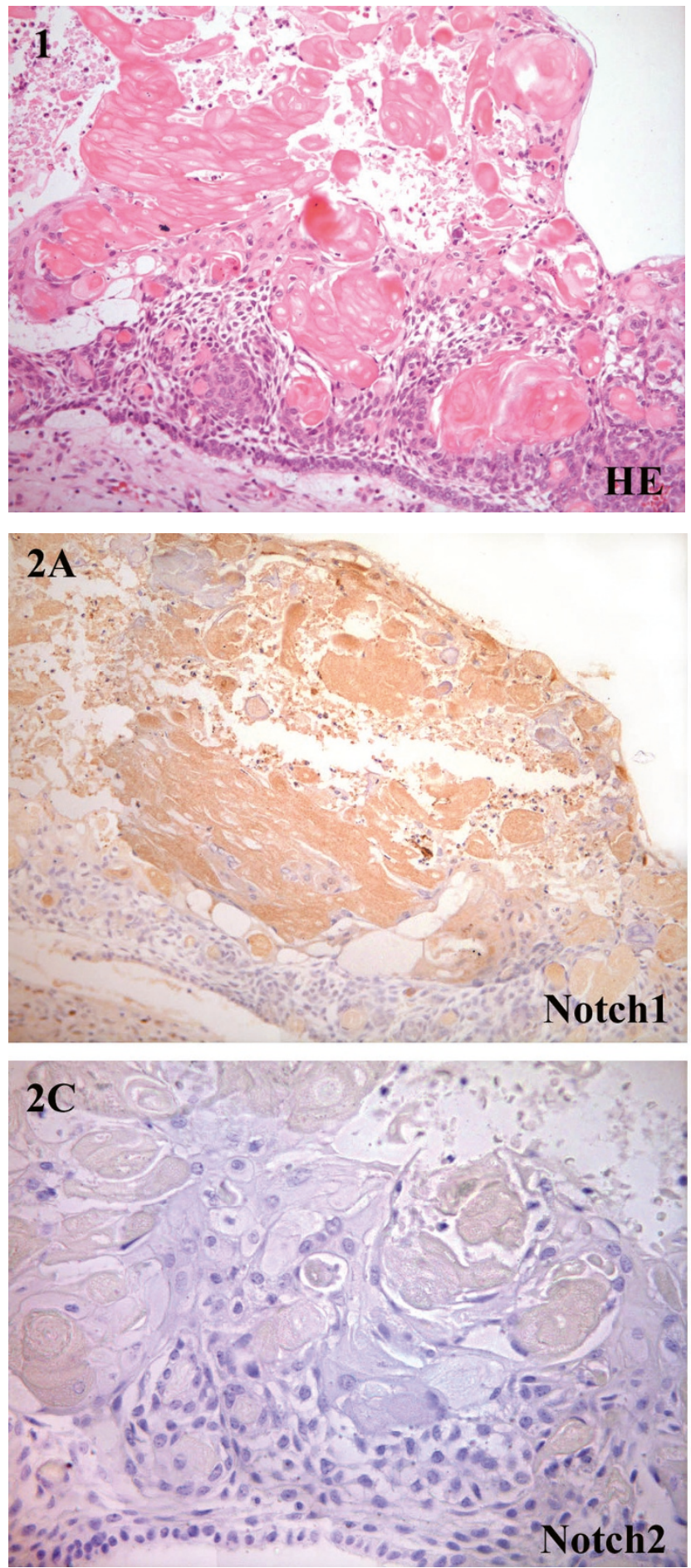

tions were systematically sampled and the level of expression for Notch (1-4), Jagged (1-2) and Delta1 was quantified according to the staining intensity and percentage of immunopositive tumoral components present (CCOT neoplastic epithelial cells, ghost cells and calcifications): (-) negative when none of these tumoral components are positively stained; $(+)$ mild when staining was present in focal areas $(<25 \%) ;(++)$ moderate when staining was evident in significant areas $(25-50 \%)$; and $(+++)$ strong when staining is present in extensive areas $(>50 \%)$. Stromal immunoreactivity was also assessed in a similar manner.

\section{RESULTS}

Microscopic Findings

All five cases of CCOT studied here were of the cystic type. Histological examination revealed lesional tissues composed of fibrous wall lined by ameloblastomatous epithelium characterized by basal cuboidal/low columnar pre-ameloblast-like cells and suprabasal stellate reticulum-like cells with scattered clusters of ghost
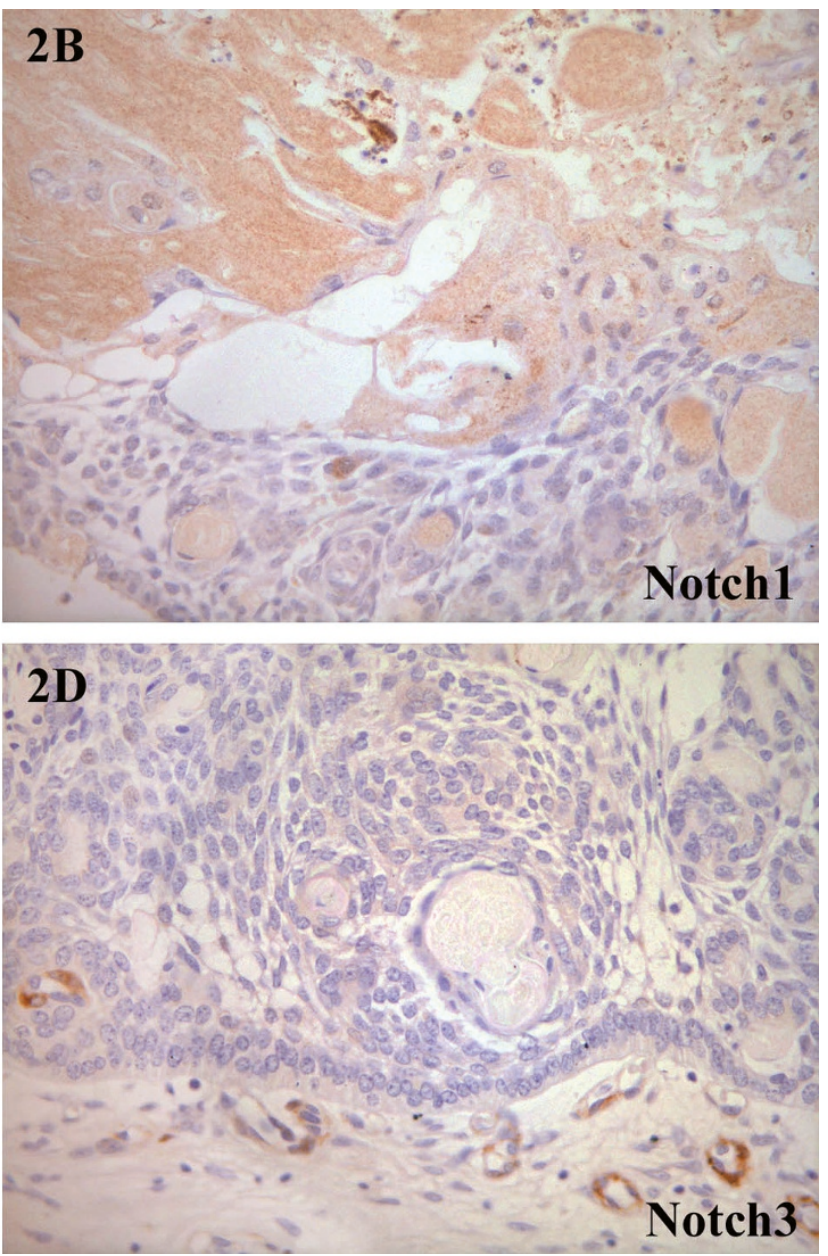

Fig. 1. Microscopic features of the calcifying cystic odontogenic tumor showing fibrous connective tissue wall lined by ameloblastomatous epithelium characterized by basal low columnar/cuboidal cells and suprabasal stellate reticulum-like cells with interspersed clusters of ghost cells (Hematoxylin \& eosin, x100).

Fig. 2. Photomicrograph of CCOT lining epithelium and ghost cells demonstrating Notch receptor proteins: A and B, Notch1: positive expression was observed in both ghost cells and tumoral epithelium; C, Notch2: all lesional components were negative; D, Notch3: weak to negative labeling of tumoral tissues. Note strong Notch3 positivity in stromal blood vessels. (A, x100, B-D, $\mathrm{x} 200)$. 

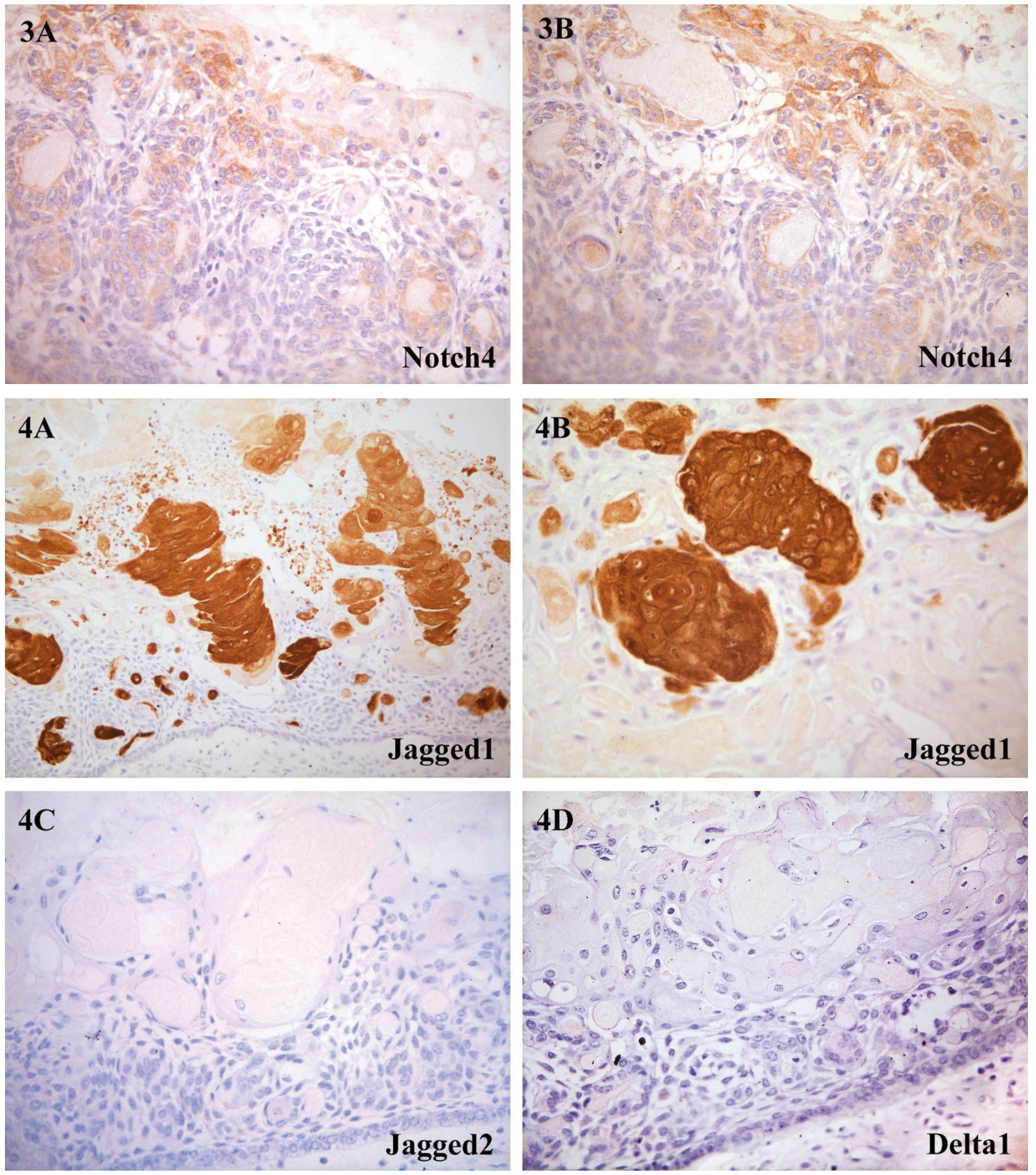

Fig. 3. Notch4: A and B, positive expression in CCOT epithelial and ghost cells; (A, x100; B, x200).

Fig. 4. Photomicrograph of CCOT lining epithelium and ghost cells demonstrating distribution of Notch ligand proteins: A and B, Jagged1: strong expression in CCOT ghost cells and mildly in squamous transitory cells and tumoral epithelium (A, x100; B, x200); C, Jagged2: no immunoreactivity was detected (x200); D, Delta1: no immunoreactivity was detected (x200).

cells and squamous transitory cells (Fig. 1). Foci of ghost cells with calcifications were also observed. Dentinoid and odontoma formation were not evident.

\section{IMMUNOHISTOCHEMICAL Findings}

Results on the semiquantitative analysis of distribution and level of positivity for Notch receptors and ligands for all five cases of CCOT are summarized in Table 2 and illustrated in Figs. 2-4. Tumoral epithelium demonstrated mild positivity for Notch1 (Fig. 2A-B), Notch4 (Fig. 3A) and Jagged1 (Fig. 3B-C) but was nonreactive for Notch2 (Fig. 2C), Notch3 (Fig. 2D), Jagged2 (Fig. 4C), and Delta1 (Fig. 4D). In those immunoreactive cells, Notch receptor and ligand protein localization was cytoplasmic and/or membranous. 
Ghost cells showed moderate labelling for Notch1 (Fig. 2A-B), mild expression for Notch4 (Fig. 3A-B) and overexpression for Jagged1 (Fig. 4A-B). Protein localization was also cytoplasmic and/or membranous. Transitory squamous cells were mildly positive for Notch1 (Fig. 2A-B), Notch4 (Fig. 3A-B) and Jagged1 (Fig. 4A-B). Both ghost cells and transitory squamous cells were not immunoreactive for Notch2 (Fig. 2C), Notch3 (Fig. 2D), Jagged2 (Fig. 4C) and Delta1 (Fig. 4D). Dentinoid and odontome were not present in all five cases of CCOT, and therefore Notch receptor and ligand distribution in these structures remained unknown. Stromal fibroblasts and blood vessels were stained variably positive.

\section{Discussion}

The calcifying odontogenic cyst is a developmental odontogenic lesion first recognized as a likely analogue of the calcifying epithelioma of Malherbe (pilomatrixoma) by Gorlin et al. in 1962 [8]. It is rare and accounts for $1-2 \%$ of all jaw cysts. Since its initial description, it became clear that the calcifying odontogenic cyst consists of two basic pathobiologic subsets: a cystic neoplasm and a solid tumor. In 2005, the World Health Organization updated its classification of odontogenic tumors [10], and reclassified the calcifying odontogenic cyst subdividing it into three distinct entities: calcifying cystic odontogenic tumor (CCOT), dentinogenic ghost cell tumor (DGCT) and ghost cell odontogenic carcinoma (GCOC) [10]. CCOT is a benign cystic odontogenic neoplasm, characterized by local invasiveness and rare recurrence following enucleation. DGCT resembles an ameloblastoma clinically and histopathologically except that large numbers of ghost cells and dysplastic dentin are present in DGCT. GCOC is a malignant odontogenic epithelial tumor characterized by a highly aggressive and recurrent biologic behavior. All five cases examined in this study belong to the CCOT category.

Other than our single CCOT case study that examined for aberrant Notch1 signaling [23], a search of the English language literature disclosed that the expression patterns of other Notch family members and their ligands in ghost cells remain unknown. The distinct histological appearance of the ghost cells in the CCOT and the critical role of Notch signaling pathway in odontogenesis led us to investigate further whether Notch signaling modulates the fate of ghost cells in this neoplasm.

Notch activation in cell fate decisions is known to operate via three distinct mechanisms: lateral inhibition, binary cell fate, and lateral induction. In the classic Notch 'lateral inhibition' cells with a given fate inhibit their neighboring cells from adopting the same fate. This means that signaling between Notch ligand and Notch receptor on an adjacent cell inhibits ligand production in the receiving cell through a negative feedback loop [24, 25]. In contrast, during binary cell fate decisions, distinct cell fates are determined by asymmetric distribution of Notch pathway components, such as the cytoplasmic Notch inhibitor, Numb. Finally, in lateral induction, which is the least well described mode of Notch action, signaling between
Notch ligand and Notch receptor on adjacent cells results in a positive feedback, which promotes ligand expression and activation of Notch on both cells. This mechanism has been suggested to propagate Notch signals through a cell-to-cell relay mechanism [25, 26]. These various modes of signaling allow Notch to perform different functions within the same tissue in a spatially and temporally regulated manner. It is believed that the role of Notch is to block differentiation by maintaining the competence of undifferentiated cells [1]. This implies that Notch expression would preferentially be found in cell types that are not terminally differentiated. During odontogenesis, the determination of cell fates in the enamel organ occurs via inhibitory interactions between adjacent dental epithelial cells. Initially, cells of the forming dental epithelium appear to constitute a developmental equivalent group in which inner enamel epithelial cells suppress differentiation in their immediate neighbours through lateral inhibition [27]. In our study, we observed that ghost cells demonstrated co-expression for Notch1 and Jagged1 indicating that there is a likelihood that signaling might occur via lateral induction between Notch ligand and Notch receptor on adjacent ghost cells resulting in a positive feedback, which promotes ligand expression and activation of Notch on both these cells [25]. On the other hand, the adjacent tumoral epithelium only weakly expressed these molecules, and these observations suggest that probably Notch signal activation in ghost cells exerts a lateral inhibitory effect on the neigboring tumoral epithelium blocking them from adopting the same cell fate. Absence of Notch members (Notch2, and 3) and ligands (Jagged 2 and Delta1) in both the ghost cells and tumoral epithelium implies that these molecules most likely play little or no role in the cytological differentiation and proliferation of this neoplasm.Notch3 positivity observed in the stromal blood vessels of CCOT indicate that Notch receptors and ligands are generated by different inductive mechanisms.

In summary, this study investigated the role of aberrant Notch signaling in ghost cell fate in five cases of CCOT. Our results suggest that Notch may play an oncogenic role in the tumorigenesis of CCOT where Notch1 signal activation induced by Jagged1 is necessary for specification of ghost cell fate.

Acknowledgements: This study is supported by FRGS FP028/2010A, Ministry of Higher Education, Malaysia and Grant-in-aid for Scientific Research (C) (20592349 and 23592951) from the Japan Society for the Promotion of Science.

\section{REFERENCES}

1. Artavanis-Tsakonas S, Rand MD and Lake RJ. Notch signaling: cell fate control and signal integration in development. Science 1999; 284: 770-776.

2. Leong KG and Karsan A. Recent insights into the role of Notch signaling in tumorigenesis. Blood 2006; 107: 22232233.

3. Kim J, Lee EH, Yook JI, Han JY, Yoon JH and Ellis GL. Odontogenic ghost cell carcinoma: a case report with reference to the relation between apoptosis and ghost cells. Oral Surg Oral Med Oral Pathol Oral Radiol Endod 2000; 90: e630-e635. 
4. Lucchese A, Scivetti M, Pilolli GP and Favia G. Analysis of ghost cells in calcifying cystic odontogenic tumors by confocal laser scanning microscopy. Oral Surg Oral Med Oral Pathol Oral Radiol Endod 2007; 104: 391-394.

5. Sonone A, Hande A, Chaudhary M, Bonde R, Sheorain A and Agni N. Adenoid ameloblastoma with dentinoid and ghost cells. A composite odontogenic tumour: a rare case report and review of the literature. Oral Surg Oral Med Oral Pathol Oral Radiol Endod 2011; 4: 77-81.

6. Kerebel B and Kerebel L-M. Ghost cells in complex odontoma: A light microscopic and SEM study. Oral Surg Oral Med Oral Pathol Oral Radiol Endod 1985; 59: 371 378.

7. Matsuzaka $\mathrm{K}$, Inoue $\mathrm{T}$, Nashimoto $\mathrm{M}$, Takemoto $\mathrm{K}$, Ishikawa H, Asaka M, Shimono M, Fujikawa $M$ and Noma H. A case of an ameloblastic fibro-odontoma arising from a calcifying odontogenic cyst. Bull Tokyo Dent Coll 2001; 42: 51-55.

8. Gorlin RJ, Pindborg JJ, Clausen FP and Vickers RA. The calcifying odontogenic cyst: a possible analog of the cutaneous calcifying epithelioma of Malherbe. Oral Surg Oral Med Oral Pathol 1962; 15: 1235-1243.

9. Fernandez-Miranda JC, Gardner PA, Snyderman CH, Devaney KO, Strojan P, Suárez C, Genden EM, Rinaldo A and Ferlito A. Craniopharyngioma: A pathologic, clinical, and surgical review. Head Neck 2011 doi: 10.1002/ hed.21771.

10. Barnes L, Eveson JW, Reichart PA and Sidransky D, eds. World Health Organization Classification of Tumors. Pathology and Genetics of Head and Neck Tumors. Lyon: IARC Press, 302-303, 2005

11. Siar $\mathrm{CH}$ and $\mathrm{Ng} \mathrm{KH}$. Orthokeratinized odontogenic keratocysts in Malaysians. Br J Oral Maxillofac Surg 1988; 26: 215-220.

12. Siar $\mathrm{CH}$ and $\mathrm{Ng} \mathrm{KH}$. Squamous cell carcinoma in an orthokeratinized odontogenic keratocyst. Int $\mathrm{J}$ Oral Maxillofac Surg 1987; 16: 95-98.

13. Siar $\mathrm{CH}$ and $\mathrm{Ng} \mathrm{KH}$. Combined ameloblastoma and odontogenic keratocyst or keratinizing ameloblastoma. $\mathrm{Br}$ J Oral Maxillofac Surg 1993; 31: 183-186.

14. $\mathrm{Ng} \mathrm{KH}$ and Siar $\mathrm{CH}$. Clear cell change in a calcifying odontogenic cyst. Oral Surg Oral Med Oral Pathol Oral Radiol Endod 1985; 60: 417-419.

15. Philipsen HP, Reichart PA, Siar CH, Ng KH, Lau SH, Zhang XL, Dhanuthai K, Swasdison S, Jainkittivong A, Meer S, Jivan V, Altini M, Hazarey V, Ogawa I, Takata T, Taylor AAM, Godoy H, Delgado WA, Carlos-Bregni R, Macias JFR, Matsuzaka K, Sato D, Vargas PA and Adebayo T. An updated clinical and epidemiological profile of the adenomatoid odontogenic tumor (AOT). A collaborative retrospective study. J Oral Pathol Med 2007; 36: 383-393.

16. Nagatsuka H, Siar CH, Nakano K, Tsujigiwa H, Gunduz M, Choufuku H, Lee YJ, Naito I, Sado Y, Nagai N. Differential expression of collagen IV alpha 1 to 6 chains in basement membrane of benign and malignant odontogenic tumors. Virch Arch 2002; 441: 392- 399.

17. Nakano K, Siar CH, Nagai N, Naito I, Sado Y, Nagatsuka H, Hoh C, Kurada K, Tsujigiwa H and Gunduz M. Distribution of basement membrane type IV collagen in ameloblastoma: An immunoflourescence study. J Oral Pathol Med 2002; 31: 494-499.
18. Chuah KS, Siar CH, Nakano K, Nagatsuka H, Khoo SP, $\mathrm{Ng} \mathrm{KH}$ and Kawakami T. Wingless type protein-1 (Wnt1) expression in primary conventional and unicystic ameloblastoma and their recurrent tumors. J Hard Tissue Biol 2009; 18: 63-70.

19. Siar $\mathrm{CH}$, Nakano K, Han PP, Nagatsuka H, Ng KH and Kawakami T. Differential expression of Notch receptors and their ligands in the desmoplastic ameloblastoma. J Oral Pathol Med 2010; 39: 552-558.

20. Siar CH, Nagatsuka H, Chuah KS, Rivera RS, Nakano K, $\mathrm{Ng} \mathrm{KH}$ and Kawakami T. Notch4 overexpression in ameloblastoma correlates with the solid/multicystic phenotype. Oral Surg Oral Med Oral Pathol Oral Radiol Endod 2010; 110: 224-233.

21. Siar CH, Nakano K, Ng KH, Tomida M, Nagatsuka H and Kawakami T. Squamous odontogenic tumor: A case report demonstrating immunoexpression for Notch1, 3, 4, Jagged1 and Delta1. Eur J Med Res 2010; 15: 180-184.

22. Siar CH, Chuah KS, Nakano K, Rivera RS, Tsujigiwa H, Nagatsuka $\mathrm{H}, \mathrm{Ng} \mathrm{KH}$ and Kawakami T. Immunohistochemical study of Notch signaling proteins in the calcifying epithelial odontogenic tumor (Pindborg tumor). J Hard Tissue Biol 2010; 19: 167-174.

23. Nakano K, Siar CH, Tsujigiwa $\mathrm{H}$, Nagatsuka $\mathrm{H}, \mathrm{Ng} \mathrm{KH}$ and Kawakami T. Immunohistochemical observation of Notch signaling in a case of calcifying cystic odontogenic tumor. J Hard Tissue Biol 2010; 19: 147-152.

24. Bray S. Notch signaling in Drosophila: three ways to use a pathway. Semin Cell Dev Biol 1998; 9: 591-597.

25. Chitnis AB. The role of Notch in lateral inhibition and cell fate specification. Mol Cell Neurosci 1995; 6: 311321.

26. Ross DA and Kadesch T. Consequences of Notch-mediated induction of Jagged1. Exp Cell Res 2004; 296: 173182.

27. Mitsiadis TA, Lardelli M, Lendahl U and Thesleff I. Expression of Notch 1, 2 and 3 is regulated by epithelialmesenchymal interactions and retinoic acid in the developing mouse tooth and associated with determination of ameloblast cell fate. J Cell Biol 1995; 130: 407-418.

Received: June 7, 2011 / Accepted: July 7, 2011

Address for correspondence:

Dr. Toshiyuki Kawakami, PhD, Professor,

Hard Tissue Pathology Unit,

Matsumoto Dental University Graduate School of

Oral Medicine,

1780 Hirooka-Gobara,

Shiojiri 399-0781,

Japan

Phone: +81-263-51-2035

Fax: +81-263-51-2035

E-mail: kawakami@po.mdu.ac.jp 